earlier time when mythological precepts held sway. Hübner makes it clear that in those times the fact itself was expressed in terms of mythological precepts, and so was experienced in a very different way to that which is now common (e.g. art and the sacred were sensed as one and indivisible). This account of the nature of the difference is such that $I$, for one, was able for the first time to appreciate the significance of the mythological approach to life as a whole, and to see that within it everything is at least as consistent as it is in our approach through science and technology.

Of course, Hübner does not expect us to return to a mythological world. However, he does suggest that sooner or later the world of science and technology will have to give way to a new world with a very different system-ensemble of precept. In this way, he indicates that the problems of our present scientific and technological world (e.g. pollution, destruction of the environment, creation of a meaningless way of life and so on) may dissolve away. On the other hand, within our own system, it is by now becoming evident that these problems cannot really be solved in any fundamental sense.

Thus far I have dealt with only some of the main points treated in the book. There are further important criticisms of a number of scientific and philosophical theories in it, as well as a carefully developed historical account of how Kepler came to discover his elliptical orbits. This account not only illustrates Hübner's theses concerning the role of systemsensembles. It also serves to raise the point that had Kepler followed Popper's judicative precepts on falsifiability, he would have dropped his work long before it could have reached a successful conclusion. Thus, he argues, Popper's falsifiability criterion cannot be universally valid.

Critique of Scientific Reason makes a new and original contribution to the understanding of how the development of knowledge actually takes place. Doubtless, though, it contains features that can be criticized. For example, while Hubner claims that his own ideas are not inseparably based on precepts (as yet unstated), it is by no means clear this is actually the case. It is to be expected that some of those who have been criticized by Hubner will make rejoinders that further serve to show the limits of his work. For the present, I would only add that just because it shows the boundaries of scientific reason, his approach tends to make scientific research seem less significant and thus may tend to damp the passion needed for great and original scientific discoveries. Nevertheless, it must be said that on the whole this book deserves very serious consideration, especially by scientists and philosophers.

David Bohm is Emeritus Professor of Theoretical Physics at Birkbeck College, University of London.

\section{Concepts of quantum theory}

\section{Hans Primas}

Quantum Theory and Measurement.

Edited by John Archibald Wheeler and Wojciech Hubert Zurek.

Princeton University Press: 1984. Pp.811. Hbk \$75, f69.30; pbk \$19.50, £13.90.

WHAT is quantum mechanics about? Is it about individual systems or about statistical ensembles? Is it about objectively existing entities, or about the results of measurements or about the sensations of the observers?

There is no general agreement, and a reference book containing the most important papers on the conceptual problems of the subject has been needed for a long time. In Quantum Theory and Measurement, John Wheeler and Wojciech Zurek have tried to satisfy this need. They have selected forty-nine papers to illuminate the meaning of quantum theory as it is disclosed in the measurement process, among them the key papers of the magnificent Bohr-Einstein dialogue, the famous paper by Einstein, Podolsky and Rosen, and Schrödinger's cat paradox (in an English translation). Most welcome are the hitherto unpublished lecture notes by Eugene Wigner on the interpretation of quantum mechanics, as well as a translation of London and Bauer's classic "The Theory of Observation in Quantum Mechanics". A pleasant surprise are the papers stressing the engineering aspects of quantum measurements, specifically dealing with optical channels, quantum noise and non-demolition measurements.

Why is the theory of measurements in quantum theory so controversial? Perhaps it is because our textbooks have uncritically adopted the pragmatic rules that working physicists required in the hectic time around 1928 in order to be able to use the new but yet incompletely developed quantum mechanics. In 1927, John von Neumann formalized not only the unitary time evolution given by the Schrödinger equation but also a second kind of state change which he supposed to occur during a measurement. This so-called projection postulate involves a projection of the state vector onto a subspace determined by the outcome of the measurement and is commonly referred to as the "collapse of the wave packet". Within the traditional formalism this collapse of the wave packet cannot be described by the Schrodinger equation. This situation gave rise to severe philosophical disagreements and wild speculations. Even mind and consciousness have been evoked as a way out of the alleged paradoxes of quantum measurements. Since the view that quantum events do not exist in the absence of some observing mind is not to everybody's liking, many disquieted physicists abandoned the view that the task of science is to describe nature. One formally admissible but rather cheap way out of this dilemma is to adopt a very narrow-minded positivistic or operationalistic viewpoint, claiming that all a theory can do is to describe and predict the results of measurements (that is, to adopt an "epistemic interpretation"). From this point of view the state vector is just a calculational tool, it does not represent reality. In its most extreme variant, the referent of the theory is the mind of the observer.

All the so-called paradoxes of quantum measurements depend crucially on the fiction of a "measurement of the first kind" (a measurement which is supposed to be instantaneous, repeatable and give sharp values). Faced with the strange consequences of the projection postulate, it is astonishing that so often the existence of measurements of the first kind is taken for granted. All actual measuring processes we really understand today (nuclear magnetic resonance experiments, for example), are certainly not measurements of this type, and they can be discussed without the projection postulate. In contradistinction, a full theoretical account of the traditional two-slit experiment is extremely complicated and out of the reach of our present abilities. Its consideration in terms of the collapse of the wave packet has only the status of a rule of thumb which should not be taken too seriously.

As to this book, it is in my view most unfortunate that the editors have not concerned themselves with modern developments. Obvious omissions are the proof that in quantum mechanics repeatable measurements can only exist for observables with a discrete spectrum (that is, the projection postulate is irrelevant for the most important observables; Davies and Lewis, 1970; Srinivas, 1980); Holevo's discussion of optimal quantum measurements (solving the old problems of the joint measurement of incompatible observables and the measurement of the time parameter); the generalization of the unitary time evolution to completely positive dynamical semigroups (which settles many questions of irreversibility in quantum mechanics); and the logical possibility that the referent of quantum mechanics is objectively existing individual systems (i.e. an "ontic interpretation" in the sense of Scheibe, 1964).

Every theoretical discussion of a realistic laboratory experiment is a difficult undertaking. An axiomatic approach starting with some postulates about measurements (such as von Neumann's projection postulate) can never do justice to the complex nature of actual experiments. From a modern point of view, the measurement problem of quantum mechanics is a problem of the interaction of quantum systems with classical systems. A classical system is characterized by a Boolean logical 
structure (or, equivalently, by a commutative algebra of observables). The essential difference between classical systems and quantum systems is not the occurrence of Planck's constant of action (classical systems may very well depend on Planck's constant) but the fact that quantum systems are entangled by Einstein-Podolsky-Rosen correlations. This holistic feature of quantum mechanics may be difficult to grasp for a scientist educated in the classical Cartesian tradition. Thirteen well-selected papers of this collection document the experimental reality of the holistic non-separability of quantum systems.

In order to understand measurements, we must understand the existence of classical subsystems in a quantum world. That is the main problem and a tough one. If it is solved, a measurement can be described by a channel (in the sense of Umegaki) which transfers the state of the quantum object to a state of the classical output system. Much research in this field has been done in the past decades and many new important results are known. None of them is mentioned in the book. Equally disappointing are the commentaries. For example, in their "Guide to Some Further Literature" the editors say that Gleason's theorem "simplified the axiomatics of quantum mechanics". Such a phrasing is grossly misleading: Gleason's theorem is a conceptually important result explaining why linear functionals can be used to

\section{Mechanics of physics}

\section{Abraham Pais}

The Quantum World.

By J.C. Polkinghorne.

Longman: 1984. Pp.100. Hbk

£7.95, \$14.95.

THE Curate of St Michael and All Angels in Bedminster is an interesting man. He knows that "science has achieved its success by restricting itself to a certain type of inquiry", and he was elected FRS for contributing to this success. He knows too that there are other issues which are essential for a grasp of our World but which are beyond the ken of science. "God is not to be put to the test."

These sayings of the Curate, culled from his recent article in the London Times, made me turn to his new book, The Quantum World, with the confidence that it would not serve up that blend of the material and the mystic which of late seems so palatable to so many. I was not disappointed. This book is short and lucid. It demands thinking but no knowledge of higher mathematics. Its language is simple. Having been reared on the principle that it is not nice to use eight-letter words in public, I am happy to report that the word ontology occurs only once. Formulae are describe quantum states. Another example of the editors' disregard of present research is to do with the irreversible behaviour of some quantum systems. The reprinted papers deal with ergodic properties and are certainly of historic interest, but helpful comments are lacking. I think it should have been the editors' duty to inform the non-specialist why ergodic properties alone are irrelevant and why one has to require stronger mixing properties.

In an anthology of this sort, some arbitrary decisions are unavoidable. But still it is hard to understand why the editors decided not to reproduce the thoughtful and masterly "Quantum Physics and Philosophy, Causality and Complementarity" by Bohr (1958) and "On the Interpretation of Quantum Mechanics" by Fock (1957).

Although the many omissions may be regretted, this set of papers makes fascinating reading. The editors expect that their collection can be used as a source book for a seminar on the subject. The historical aspects are certainly well covered so it is, indeed, an obvious starting point for those entering the field. But it far from represents the state of the art, and no reader should suppose that the book will allow him to catch up with modern developments.

Hans Primas is Professor in the Laboratorium für Physikalische Chemie at ETH-Zentrum, Zürich.

given here and there but mainly to show what they look like. Nine brief appendices introduce the reader to more arcane subjects including a half-page derivation of John Bell's celebrated criterion for the validity of quantum mechanics which has recently been subjected to stringent laboratory tests. (No surprises: quantum mechanics works.) A helpful glossary is also included. Princes and Poppers alike will derive benefit from this handsomely produced volume.

"Professional practitioners of quantum mechanics can calculate away without worrying about the outcome of the debates about the foundations of their subject" (p.16) which centre on the question: is it necessary to include a specification of the experimental arrangement in defining what we mean by a physical phenomenon? "No", says the classical mechanic who uses the code words: objective reality. "Yes", says the quantum mechanic whose code word is complementarity and who bases his argument on the ineluctable indeterminacy principle. After giving a clear account of the transition from the classical to the quantum era, the author guides us through the quantum arguments by the fitting device of a tour through thought experiments which illuminate this subtle issue. One such experiment is that of Einstein, Podolsky and Rosen. I regret that the author follows others by referring to the
EPR result as a paradox. The EPR paper, logically impeccable, merely shows that objective reality and complementarity cannot both be true. Einstein never called it a paradox. Nor was he "wistful[ly] longing after a determinate hidden variable theory" (p.73). He never referred to "hidden variables" in his writings and in fact of ten called them a cheap way out.

In addressing these questions of principle, care with language is of the essence. I cannot subscribe to the author's statement (p.60): "Measurement involves an intervention by our everyday world into

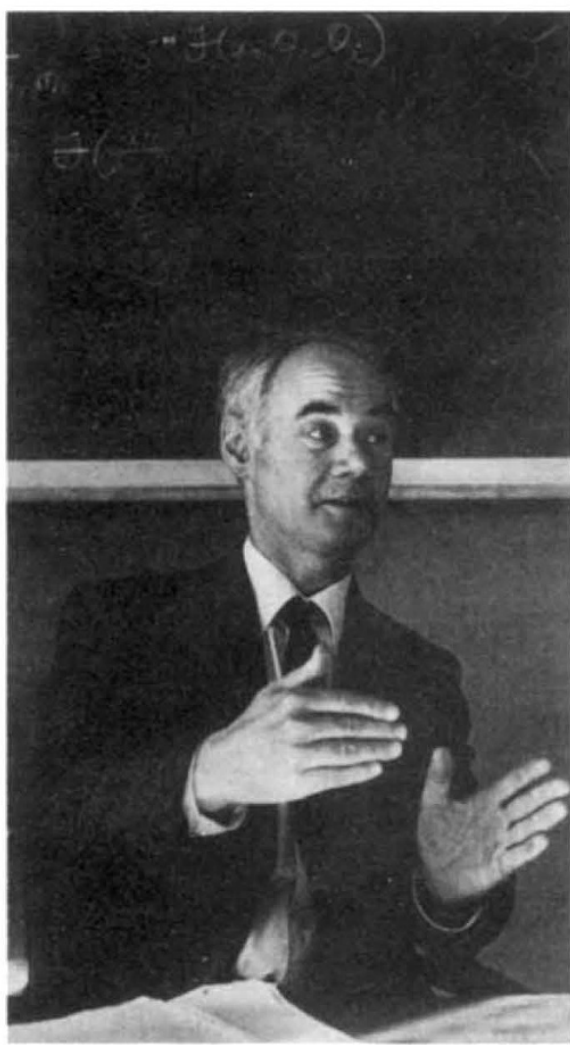

John Polkinghorne - "testimony to the fact that physics is necessary but not sufficient for salvation, that science and religion can coexist in harmonious complementarity".

the quantum world". I would rather say with Bohr: "There is no quantum world

It is wrong to think that the task of physics is to find out how nature is"' (p.79). May we then not ask what the World is? Of course we may, but here physics "restricting itself to a certain type of inquiry", cannot give answers. The author's life as well as his oeuvre, especially this lovely little book, bear testimony to the fact that physics is necessary but not sufficient for salvation, that science and religion can coexist in harmonious complementarity. He would agree, I think, with the quantum mechanic's reply to Einstein's assertion that God does not play dice: how do you know?

Abraham Pais is Professor of Physics at the Rockefeller University, New York. His biography of Albert Einstein. 'Subtle is the Lord first published in 1982, has recently been issued in paperback by Oxford University Press. 